\title{
Risk Factors for Seizures Among Young Children Monitored With Continuous Electroencephalography in Intensive Care Unit: A Retrospective Study
}

\begin{abstract}
Jan Vlachy ${ }^{1}$, Mingyoung Jo ${ }^{2}$, Qing Li ${ }^{3}$, Turgay Ayer ${ }^{1}$, Pinar Keskinocak ${ }^{1}$, Julie Swann ${ }^{4}$, Larry Olson ${ }^{2,5}$ and Atul Vats ${ }^{2,6 *}$

${ }^{1}$ School of Industrial and Systems Engineering, Georgia Institute of Technology, Atlanta, GA, United States, ${ }^{2}$ Children's Healthcare of Atlanta, Atlanta, GA, United States, ${ }^{3}$ Johns Hopkins Applied Physics Lab, Baltimore, MD, United States, ${ }^{4}$ Department of Industrial and Systems Engineering, North Carolina State University, Raleigh, NC, United States, ${ }^{5}$ Departments of Pediatrics and Neurology, Emory University, Atlanta, GA, United States, ${ }^{6}$ Department of Pediatrics, Emory University, Atlanta, GA, United States
\end{abstract}

Objective: $C E E G$ is an emerging technology for which there are no clear guidelines for patient selection or length of monitoring. The purpose of this study was to identify subgroups of pediatric patients with high incidence of seizures.

Study Design: We conducted a retrospective study on 517 children monitored by cEEG in the intensive care unit (ICU) of a children's hospital. The children were stratified using an age threshold selection method. Using regression modeling, we analyzed significant risk factors for increased seizure risk in younger and older children. Using two alternative correction procedures, we also considered a relevant comparison group to mitigate selection bias and to provide a perspective for our findings.

Results: We discovered an approximate risk threshold of 14 months: below this threshold, the seizure risk increases dramatically. The older children had an overall seizure rate of $18 \%$, and previous seizures were the only significant risk factor. In contrast, the younger children had an overall seizure rate of $45 \%$, and the seizures were significantly associated with hypoxic-ischemic encephalopathy ( $\mathrm{HIE} ; p=0.007$ ), intracranial hemorrhage ( $\mathrm{ICH} ; p=0.005)$, and central nervous system $(\mathrm{CNS})$ infection $(p=0.02)$. Children with $\mathrm{HIE}, \mathrm{ICH}$, or CNS infection accounted for $61 \%$ of all seizure patients diagnosed through cEEG under 14 months.

Conclusions: An extremely high incidence of seizures prevails among critically ill children under 14 months, particularly those with $\mathrm{HIE}, \mathrm{ICH}$, or CNS infection.

Keywords: continuous EEG, pediatric, non-convulsive, intensive care, brain injury

\section{INTRODUCTION}

Nonconvulsive seizures in intensive care unit (ICU) patients do not manifest convulsive activity, but they can be detected through continuous electroencephalography (cEEG) $(1,2)$. These seizures are more prevalent among critically-ill children than previously thought (2). Furthermore, growing evidence suggests that non-convulsive seizures in ICU are associated with worse patient 
outcomes (3-5). To detect non-convulsive seizures, monitoring patients at high seizure risk with cEEG is crucial. However, interpreting cEEG is very labor intensive and requires substantial investment in equipment, technician support, and network infrastructure (5). Therefore, it is not feasible to universally monitor all ICU children with neurological conditions, which motivated us to systematically identify high-risk conditions that warrant monitoring.

Certain groups of children have already emerged as having a higher risk of seizures in several studies (2, 6-12); however, these findings remain limited because of small sample sizes. A multicenter study (13) attempted to address sample size, however, it included few young children and children with acute structural diagnoses. There is a consensus statement for monitoring children, a guideline for neonates $(5,14)$ and a risk prediction model for children (15). Our objective in this large single center study is to identify high-risk conditions in a large cohort of young children.

\section{MATERIALS AND METHODS}

\section{Setting}

The retrospectively-reviewed study population consisted of all children who underwent cEEG monitoring at the Egleston campus of Children's Healthcare of Atlanta (Children's) from June 2010 to August 2013.

The Egleston campus of Children's is a 255-bed free-standing children's hospital providing quaternary care. For these children, the attending physician is responsible for ordering monitoring. In the hospital, cEEG monitoring occurs in the following units: a 30-bed medical-surgical pediatric intensive care unit (PICU), a 28-bed cardiac intensive care unit (CICU), and a 45-bed neonatal intensive care unit (NICU).

The results of continuous EEGs were recorded digitally using 21 scalp electrodes, affixed with paste or collodion adhesive, with a bedside computer networked to a central EEG server. There were no constraints placed on the number of cases placed on EEG at any one time. EEG technician coverage was available $24 \mathrm{~h}$ per day. EEG studies were reviewed by the attending neurophysiologist at least 3 times per $24 \mathrm{~h}$ but typically every $2-4 \mathrm{~h}$ or as necessary when seizures were active. The duration of monitoring was determined by the attending physician. Often, the duration would be between 24 and $48 \mathrm{~h}$ according to the common practice, but sometimes the duration would be much longer.

\section{Participants}

The retrospective population consisted of all children who underwent cEEG from June 2010 to August 2013. This patient population was of very high acuity [for instance, the PICU

\footnotetext{
Abbreviations: cEEG, continuous electroencephalography; Children's, Children's Healthcare of Atlanta; CICU, cardiac intensive care unit; CNS, central nervous system; EEG, electroencephalography; HIE, hypoxic-ischemic encephalopathy; $\mathrm{ICH}$, intracranial hemorrhage; ICU, intensive care unit; IPWE, inverse probability weighted estimation; IQR, inter-quartile range; NICU, neonatal intensive care unit; OR, odds ratio; PICU, pediatric intensive care unit; VPS, Virtual Pediatric Systems, LLC.
}

was in the top $25 \%$ of participants in the Virtual Pediatric Systems database (16)]. To account for possible selection bias (as discussed in more detail later), we also compared this population with a more representative control sample [utilizing internal data from Children's Virtual Pediatric Systems, LLC (VPS) (16)] consisting of all PICU patients with neurological conditions at the Egleston campus of Children's from December 2010 to May 2014. Since most children in the control sample had not been monitored, this sample did not provide information on seizures, but it did contain conditions and age. Recorded conditions and ages then enabled a comparison of the control and monitored samples.

\section{Objectives}

The primary objective was to identify subgroups of pediatric patients with high incidence of seizures. We defined such subgroups based on the indication for monitoring (also referred to as "diagnosis" in the following). New monitoring cases were identified from the neurophysiology database within 5 days from the start of the cEEG, on average within $24 \mathrm{~h}$. The indication for the cEEG was abstracted from the chart at the time the study was ordered. Indications and patient characteristics included previous seizures, witnessed definite acute seizures, age, location, concerns of altered mental status, central nervous system (CNS) infection, hypoxicischemic encephalopathy (HIE), cerebrovascular accident (CVA), intracranial hemorrhage ( $\mathrm{ICH})$, acute trauma, toxic/metabolic encephalopathy, tumor, congenital malformation, spells (acute behavioral or physiological changes), or other reasons. All indications were recorded as binary variables if they were cited as reasons for initiating cEEG monitoring. A patient could have multiple indications (e.g., ICH and trauma).

\section{Outcomes}

The primary outcome of the study was the incidence of any seizure. In the following, we describe how this outcome was measured.

After monitoring was completed, the duration of monitoring and time to first seizure and last seizure were determined by chart review of the neurophysiology reports. These studies were read by several different EEG readers who were neurologists trained in formal Epilepsy and EEG fellowships and had 725 years of experience in reading continuous EEG monitoring. It was also determined if "spells" occurred of the same type as the initial concerns, and whether or not they were seizures. If seizures had clear clinical behavioral change which could be recognized at bedside or on video, they were recorded as (clinical, "convulsive") "seizures," otherwise as non-convulsive seizures. When electrographic seizures occupied $50 \%$ or more of the EEG for longer than $30 \mathrm{~min}$, they were labeled as "frequently repetitive seizures," in order to identify a subgroup with extremely frequent, persistent, non-convulsive seizures. For purposes of this study, "seizures" refers to all three of these types of events. Electrographic seizures were considered separate from periodic or other rhythmic patterns if they had durations longer than $10 \mathrm{~s}$, and evolved in frequency or field (not amplitude alone). Inter-rater reliability in seizure recognition was not explicitly 
examined, but notes were kept regarding any disparities. These were unusual and typically minor.

All three types of seizures (clinical, non-convulsive, and frequently repetitive) counted as a seizure in the statistical models. Seizure incidence served as the outcome variable in the models that follow while neurological conditions described in Objectives jointly served as explanatory variables. These variables were recorded consistently, with no missing observations.

\section{Sample Size Estimation}

This was a retrospective study and no prior sample size determination had been conducted.

\section{Statistical Analysis}

In the following sections, we describe the methods that we used for the age threshold selection, seizure risk modeling, and selection bias correction. We conducted the analyses using the statistical software R (17). Unless stated otherwise, we report the statistical significance at the level of $\alpha=0.05$.

\section{Age Threshold Selection}

The literature indicates that infants and older children substantially differ in observed seizure incidence $(9,13)$ which suggests that for each of the two groups, different risk factors may play a role. Since the exact age where such a risk transition would occur is unknown, we stratified patients as infants or older children according to their observed seizure incidence using a statistical approach suggested by Ross and Tasoulis (18). This methodology assumes that children below and above a certain threshold age have unknown but different seizure probabilities. Then, a value of a statistic based on Fisher's exact test (19) determines the most appropriate age threshold to separate younger and older children. After this stratification, we conducted remaining analyses on each age group separately.

\section{Seizure Risk Modeling}

We used multivariate logistic regression (20) to estimate the seizure risk. The outcome variable was the occurrence of any seizures during EEG monitoring, but we also tested the outcome variable being non-convulsive seizures only. The multivariate model is stratified by age and includes as predictors all diagnoses that occurred in a sufficient number of patients. We do not further reduce the number of predictors because our sample size is sufficient, which is considered a good practice when the purpose of the model is effect estimation (21). We include results from univariate models for each of the diagnoses. Goodnessof-fit of the multivariate model was tested using the HosmerLemeshow test (19). Next, to understand the effect of multiple conditions, we tested if seizure risk was magnified for children with multiple neurological conditions. Furthermore, to assess sensitivity to the choice of the age threshold, we reran the analysis with thresholds of 12 and 24 months. Also, since the control sample is drawn from PICU only, we reran the analysis on the subset of PICU children to see if the results qualitatively change. Further, since neonates (children under 1 month) have been studied in the literature separately and may differ from other young children, we conducted additional analyses separately for children under 1 month and between one and 14 months. Finally, we also considered two alternative models where we also included as covariates (a) having any other, infrequent diagnoses or (b) the type of ICU.

\section{Selection Bias Correction}

The ICU physicians ordered monitoring at their discretion which inherently might have induced a selection bias in our sample. We would expect that children were more likely to receive monitoring if perceived as being at high risk. Therefore, the children in our sample may exhibit higher prevalence of seizures than the general PICU neurological population. To partially account for this possible bias, we compared the monitored children to the ones from the control sample. We evaluated the selection bias using two different approaches, the inverse probability weighted estimation (IPWE) and the second method by Huang et al. (22). Both methods are described in more detail in Appendix 1 in Supplementary Material.

\section{RESULTS}

The dataset contained 517 pediatric monitored patients. Among the 150 patients who experienced seizures during cEEG monitoring, 68 had exclusively non-convulsive seizures. Because cerebrovascular accident, congenital malformation, toxic/metabolic encephalopathy, and tumor were each recorded in $<10$ patients in the younger age group, we excluded these conditions from the main model but kept them grouped within "Other diagnosis" in one of the supplementary models.

The age threshold selection yielded 14 months as the age cutoff. Of 517 children, 215 were 14 months or younger and 302 of them were older than 14 months, with median age of 24 months (IQR $=3$ months -9 years). Most of the monitored children were in PICU (136 younger than 14 months and 287 older than 14 months). The monitoring duration was $<12 \mathrm{~h}$ in $9 \%(n=49)$ of patients, $12-24 \mathrm{~h}$ in $26 \%(n=134), 24-48$ in $31 \%$ ( $n=162), 48-72$ in $13 \%(n=67)$ and more than 72 in $20 \%$ $(n=105)$. Among the children with seizures, the first seizure (after the start of monitoring) was observed in 57\% within the first hour, in $22 \%$ within $1-6 \mathrm{~h}$, in $7 \%$ within $6-12 \mathrm{~h}$, in $6 \%$ within $12-24 \mathrm{~h}$, and in $7 \%$ in more than $24 \mathrm{~h}$. The first seizure time was missing in 15 children. Further summary statistics are provided in Table 1. Detailed breakdowns by departments (PICU/NICU/CICU) are available in Table 2. Six children not listed in the table were in Technology-dependent ICU (a lower acuity pediatric ward) and not included in the breakdown.

\section{Seizure Risk}

For children over 14 months, only previous seizures were associated in the multivariate model with significantly higher seizure risk $(p<0.001)$. Therefore, we focus on results for children under 14 months. Estimates and confidence intervals from the multivariate logistic regression model applied to children under 14 are presented in Table 3A. In this model, $\mathrm{ICH}, \mathrm{HIE}$, and infection were significantly associated with the increased risk of seizures. The Hosmer-Lemeshow test did not reveal a statistically significant lack of fit $(p=0.75)$, which 
TABLE 1 | The characteristics of monitored children (included are all children receiving CEEG during the study period; stratified by age group: younger or older than 14 months).

\begin{tabular}{|c|c|c|c|}
\hline & \multicolumn{3}{|c|}{ Age group } \\
\hline & All & $\begin{array}{l}\text { Younger } \\
\text { than } 14 \mathrm{~m}\end{array}$ & $\begin{array}{l}\text { Older than } \\
14 \mathrm{~m}\end{array}$ \\
\hline Total $(N=517)$ & 517 & 215 & 302 \\
\hline \multicolumn{4}{|c|}{ SEIZURES DURING MONITORING, $\boldsymbol{n}(\%)$} \\
\hline Convulsive seizures & $80(15)$ & $48(22)$ & $32(11)$ \\
\hline Nonconvulsive seizures & $116(22)$ & $75(35)$ & $41(14)$ \\
\hline Frequently repetitive seizures & $68(13)$ & $46(21)$ & $22(7)$ \\
\hline Any seizures or status* & $150(29)$ & $97(45)$ & $53(18)$ \\
\hline No seizures/status & $367(71)$ & $118(55)$ & $249(82)$ \\
\hline \multicolumn{4}{|c|}{ INDICATIONS FOR MONITORING, $n$ (\%) } \\
\hline Prior seizures/epilepsy & $206(40)$ & $69(32)$ & $137(45)$ \\
\hline Intracranial hemorrhage $(\mathrm{ICH})$ & $77(15)$ & $47(22)$ & $30(10)$ \\
\hline $\begin{array}{l}\text { Hypoxic-ischemic } \\
\text { encephalopathy (HIE) }\end{array}$ & $90(17)$ & $48(22)$ & $42(14)$ \\
\hline Head trauma & $53(10)$ & $24(11)$ & $29(10)$ \\
\hline $\begin{array}{l}\text { Infection (encephalitis or } \\
\text { meningitis) }\end{array}$ & $36(7)$ & $15(7)$ & $21(7)$ \\
\hline Coma or obtundation & $98(19)$ & $17(8)$ & $81(27)$ \\
\hline Spells & $170(33)$ & $93(43)$ & $77(25)$ \\
\hline Cerebrovascular accident & $15(3)$ & $5(2)$ & $10(3)$ \\
\hline Congenital malformation & $9(2)$ & $6(3)$ & $3(1)$ \\
\hline $\begin{array}{l}\text { Toxic or metabolic } \\
\text { encephalopathy }\end{array}$ & $32(6)$ & $8(4)$ & $24(8)$ \\
\hline Tumor & $27(5)$ & $3(1)$ & $24(8)$ \\
\hline $\begin{array}{l}\text { Monitoring time }(\mathrm{h}), \text { median } \\
(\mathrm{IQR})^{\star \star}\end{array}$ & $32(20-64)$ & $38(22-71)$ & $29(19-53)$ \\
\hline $\begin{array}{l}\text { Time to first seizure }(h), \text { median } \\
(I Q R)^{\star \star \star}\end{array}$ & $0.5(0.2-4.3)$ & $0.5(0.0-4.3)$ & $0.5(0.0-4.2)$ \\
\hline
\end{tabular}

$I Q R$, Interquartile range in the format (1st quartile-3rd quartile).

*Including convulsive seizures, non-convulsive seizures, and non-convulsive status epilepticus. Some patients may have two or three of these (e.g., both convulsive and non-convulsive seizures).

${ }^{*}$ Monitoring time was unavailable for 31 children.

${ }^{\star \star \star} A$ mong children with seizures. Time to first seizure was unavailable for 15 children.

suggested that our model is well calibrated. After refitting the model for children under 12 or under 24 months, ICH, HIE, and infection remained statistically significant, even though previous seizures were also significant at $\alpha=0.05$ level in both cases $(p=0.02$ and 0.05 , respectively). Similarly, the model counting only non-convulsive seizures resulted in the same set of significant predictors. Having multiple neurological conditions was associated with a higher seizure risk in children under 14 months [unadjusted odds ratio $=3.5$; confidence interval (2.0, 6.3)]. The results were qualitatively similar when we reran the analysis on the subsample of PICU children. When the model was run separately for children under 1 month $(N=86)$ and the ones between one and 14 months $(N=129)$, the previously significant regression coefficients retained the same direction in both subsets. In the model that included a covariate for "Other diagnoses," this covariate was not statistically significant. The model that controlled for the ICU type exhibited a lower risk
TABLE 2 | The characteristics of monitored children, stratified by department (PICU/NICU/CICU) [excluded are 6 children monitored in Technology-dependent ICU (TICU)].

\begin{tabular}{|c|c|c|c|}
\hline & \multicolumn{3}{|c|}{ Department } \\
\hline & PICU & NICU & CICU \\
\hline Total $(N=511)$ & 423 & 45 & 43 \\
\hline Under $14 \mathrm{~m}$ & 136 & 45 & 31 \\
\hline \multicolumn{4}{|c|}{ SEIZURES DURING MONITORING, $\boldsymbol{n}(\%)$} \\
\hline Convulsive seizures & $64(15)$ & $9(20)$ & $6(14)$ \\
\hline Nonconvulsive seizures & $96(23)$ & $11(24)$ & $8(19)$ \\
\hline Frequently repetitive seizures & $60(14)$ & $5(11)$ & $3(7)$ \\
\hline Any seizures or status* & $122(29)$ & $16(36)$ & $10(23)$ \\
\hline No seizures/status & $301(81)$ & $29(64)$ & $33(77)$ \\
\hline \multicolumn{4}{|c|}{ INDICATION FOR MONITORING, $\boldsymbol{n}(\%)$} \\
\hline Prior seizures/epilepsy & $188(44)$ & $10(22)$ & $6(14)$ \\
\hline Intracranial hemorrhage $(\mathrm{ICH})$ & $59(14)$ & $9(20)$ & $9(21)$ \\
\hline $\begin{array}{l}\text { Hypoxic-ischemic } \\
\text { encephalopathy (HIE) }\end{array}$ & $67(16)$ & $7(16)$ & $16(37)$ \\
\hline Head trauma & $52(12)$ & $1(2)$ & $0(0)$ \\
\hline $\begin{array}{l}\text { Infection (encephalitis or } \\
\text { meningitis) }\end{array}$ & $33(8)$ & $1(2)$ & $1(2)$ \\
\hline Coma or obtundation & $91(22)$ & $1(2)$ & $5(12)$ \\
\hline Spells & $122(29)$ & $29(64)$ & $14(33)$ \\
\hline Cerebrovascular accident & $13(3)$ & $0(0)$ & $2(5)$ \\
\hline Congenital malformation & $6(1)$ & $1(2)$ & $1(2)$ \\
\hline $\begin{array}{l}\text { Toxic or metabolic } \\
\text { encephalopathy }\end{array}$ & $30(7)$ & $2(4)$ & $0(0)$ \\
\hline Tumor & $26(6)$ & $0(0)$ & $0(0)$ \\
\hline $\begin{array}{l}\text { Monitoring time (h), median } \\
(\mathrm{IQR})^{\star \star}\end{array}$ & 35 (21-68) & $25(20-44)$ & $22(15-43)$ \\
\hline $\begin{array}{l}\text { Time to first seizure }(\mathrm{h}) \text {, median } \\
(\mathrm{IQR})^{\star \star \star}\end{array}$ & $0.6(0.0-4.6)$ & $0.5(0.3-1.8)$ & $0.2(0.1-2.1)$ \\
\hline
\end{tabular}

$I Q R$, Interquartile range in the format (1st quartile-3rd quartile).

${ }^{*}$ Including convulsive seizures, nonconvulsive seizures, and nonconvulsive status epilepticus. Some patients may have two or three of these (e.g., both convulsive and nonconvulsive seizures).

${ }^{* *}$ Monitoring time was unavailable for 25 children in PICU, 3 in NICU, and 2 in $\mathrm{CICU}$.

${ }^{* * *}$ Among children with seizures. Time to first seizure was unavailable for 13 children in PICU and one in NICU.

for children in CICU, but the results for specific diagnoses were not substantially impacted (HIE, ICH, and infection remained statistically significant with similar point estimates). Detailed results for the last two models can be found in Table $\mathbf{3 B}$.

The results largely persisted in the selection-bias corrected models. Namely, the two correction methods that we used consistently pointed to a higher risk among children with $\mathrm{ICH}$ and HIE. Detailed results for the selection-bias corrected models can be found in the Appendix in Supplementary Material.

\section{DISCUSSION}

\section{Key Results and Interpretation}

This study reveals that the high seizure risk previously reported in neonates might also apply to infants older than 1 month, namely children below 12-24 months (our best estimate of 
TABLE 3A | Logistic regression of risk factors associated with any seizures among children under 14 months.

\begin{tabular}{|c|c|c|c|c|}
\hline \multirow[t]{2}{*}{ Factor } & \multicolumn{2}{|c|}{ Univariate analysis } & \multicolumn{2}{|c|}{ Multivariate analysis } \\
\hline & $\begin{array}{l}\text { Odds ratio } \\
\text { (95\% Cl) }\end{array}$ & $p$-value & $\begin{array}{l}\text { Odds ratio } \\
\text { (95\% Cl) }\end{array}$ & $p$-value \\
\hline Coma/obt. & $1.41(0.52,3.89)$ & 0.50 & $1.32(0.43,4.11)$ & 0.62 \\
\hline $\begin{array}{l}\text { Prior } \\
\text { seizure }\end{array}$ & $1.81(1.02,3.24)$ & $0.04^{*}$ & $1.86(0.94,3.74)$ & 0.08 \\
\hline Infection & $2.60(0.89,8.59)$ & 0.09 & $3.93(1.26,13.74)$ & $0.02^{\star}$ \\
\hline $\mathrm{ICH}$ & $3.38(1.73,6.88)$ & $<0.001^{\star \star \star}$ & $3.05(1.42,6.74)$ & $0.005^{\star \star}$ \\
\hline HIE & $2.79(1.45,5.54)$ & $0.003^{\star *}$ & $2.95(1.37,6.60)$ & $0.007^{\star \star}$ \\
\hline Trauma & $4.25(1.70,12.17)$ & $0.003^{\star \star}$ & $2.10(0.70,6.89)$ & 0.20 \\
\hline Spells & $0.39(0.22,0.68)$ & $0.001^{\star *}$ & $0.80(0.40,1.64)$ & 0.54 \\
\hline $\begin{array}{l}\text { Multiple } \\
\text { diagnoses }\end{array}$ & $3.52(2.01,6.27)$ & $<0.001^{\star \star \star}$ & & \\
\hline
\end{tabular}

TABLE 3B | Logistic regression of risk factors associated with any seizures among children under 14 months: Supplementary multivariate models.

\begin{tabular}{|c|c|c|c|c|}
\hline \multirow[t]{2}{*}{ Factor } & \multicolumn{2}{|c|}{ Include Other Dx } & \multicolumn{2}{|c|}{$\begin{array}{l}\text { Include Location } \\
\text { (baseline = PICU) }\end{array}$} \\
\hline & $\begin{array}{l}\text { Odds ratio } \\
(95 \% \mathrm{Cl})\end{array}$ & $p$-value & $\begin{array}{l}\text { Odds ratio } \\
(95 \% \mathrm{Cl})\end{array}$ & $p$-value \\
\hline Coma/obt. & $1.33(0.43,4.19)$ & 0.62 & $1.20(0.39,3.71)$ & 0.75 \\
\hline Prior seizure & $1.88(0.95,3.78)$ & 0.08 & $1.57(0.77,3.23)$ & 0.21 \\
\hline Infection & $4.16(1.32,14.70)$ & 0.02 & $3.22(1.01,11.43)$ & $0.05^{*}$ \\
\hline $\mathrm{ICH}$ & $3.11(1.44,6.91)$ & $0.004^{* \star}$ & $3.58(1.61,8.23)$ & $0.002^{\star *}$ \\
\hline HIE & $3.15(1.44,7.19)$ & $0.005^{\star \star}$ & $2.96(1.36,6.71)$ & $0.007^{\star \star}$ \\
\hline Trauma & $2.20(0.72,7.35)$ & 0.20 & $1.55(0.49,5.29)$ & 0.47 \\
\hline Spells & $0.85(0.42,1.76)$ & 0.66 & $0.73(0.35,1.52)$ & 0.40 \\
\hline Other Dx & $1.53(0.55,4.21)$ & 0.41 & & \\
\hline $\mathrm{CICU}$ & & & $0.38(0.14,0.98)$ & $0.05^{*}$ \\
\hline $\mathrm{NICU}$ & & & $0.71(0.32,1.55)$ & 0.39 \\
\hline TICU & & & $1.14(0.05,12.9)$ & 0.92 \\
\hline
\end{tabular}

${ }^{*} p<0.05 ;{ }^{* *} p<0.01$. HIE, hypoxic-ischemic encephalopathy; $\mathrm{ICH}$, intracranial hemorrhage; PICU, pediatric ICU; CICU, cardiac ICU; NICU, neonatal ICU; TICU, technology-dependent ICU. Other Dx: One or more of cerebrovascular accident, toxic/metabolic encephalopathy, tumor, or congenital malformations.

the threshold is 14 months). The critically-ill children under 14 months that have the diagnosis of HIE, ICH, or possibly meningitis or encephalitis are particularly at risk. These results complement risk factors identified in previous studies $(2,6-9,13)$ including head trauma, recent prior seizures, acute presentation of epilepsy, and younger age. Head trauma did not appear as an independent predictor in our analysis possibly due to the fact that $\mathrm{ICH}$, which is often associated with trauma, was included in the analysis. Compared to previous studies which were based on relatively smaller sample sizes (with 130 or fewer patients), our analysis with 517 patients is to our knowledge the largest single-institution study on pediatric cEEG monitoring. The only study with comparable size is a multi-center study (13) which included 550 patients. Compared with the study population in this multicenter study, our population is younger (median 24 vs. 36.5 months), and it includes a substantial number of children with acute structural neurological diagnoses such as $\mathrm{HIE}, \mathrm{ICH}$, CNS infection, and traumatic brain injury (48 vs. $24 \%$ in the multicenter study which may also suggest on average more severe patient population in our study). This allowed us to study the younger age group separately and uncover $\mathrm{HIE}, \mathrm{ICH}$, and CNS infection as risk factors for younger children, which were not found in the multicenter study.

Our dataset shows a very high rate of seizures (45\%) among younger monitored patients (under 14 months). These numbers contrast with the seizure rate of $18 \%$ observed in the monitored children over 14 months which is on par with the rates observed in adult ICU units [(23), Figure 5.1]. Even in the entire neurological PICU population under 14 months, our estimates indicate a seizure rate around $30 \%$. Higher seizure rates for younger children have been previously observed in other studies $(2,9,13)$. Our findings indicate that distinguishing younger and older age groups of children in monitoring decisions is essential.

Based on our results, monitoring all children 14 months of age or younger with ICH or HIE may be warranted. In our population, this rule would capture $53 \%$ of monitored patients with any diagnosis under 14 months for which seizures were detected. In other words, its sensitivity is 53\%. The rule would also have the positive predictive value of $62 \%$ in the monitored sample. Of course, whether these results generalize to unmonitored children is unclear. Assuming so, in our control sample, just 33\% (45 out of 136) of children under 14 months with ICH or HIE received any EEG monitoring. Among the other 91 unmonitored children, our estimates suggest that as many as 53 may actually have suffered from seizures and most of these were not clinically detected. Although more extensive monitoring may be warranted, it would require additional resources. In fact, if all these children were monitored, the total volume of EEG monitoring in PICU would have been 16\% higher (587 monitored children compared to the actual 496 monitored children). We acknowledge that there may be other reasons not to initiate monitoring in some high risk patients. For example, clinical setting and lack of brain function on exam with a dismal prognosis for survival may justify withdrawal of care without ICU EEG.

We concur with Abend et al. (13) that a multicenter prospective screening study of all children in PICU for specified cEEG indications is desirable. Until then, our study provides a better understanding of the younger patient population, which was relatively pauce in prior studies.

\section{Limitations}

The main limitation of our study is the retrospective design. Also, our study population consisted of children from only one hospital, so the acuity and diagnoses of patients may not be fully representative of general pediatric ICU population with neurological conditions. The next limitation is the lack of guidelines and recommendations regarding patient selection for cEEG monitoring. Although an institutional brain trauma 
pathway provides recommendations for antiepileptic therapy and suggests cEEG monitoring, even for these patients, the ICU physician had the ultimate authority in the decision whether to monitor. In terms of anticonvulsants, while a detailed analysis thereof was not within the scope of this study, the neurologists and intensivists were all operating within an accepted treatment protocol, so treatment approaches would be similar for all patients. Factors that influence the decision to monitor might include ICU, provider, diagnosis, or age, and could vary over time with experience with which cases are high or low risk. Furthermore, the data did not include any scores measuring the severity of illness. We attempted to address some of these selection bias limitations (in particular, the selection on observables) by using a correction method. Finally, even though we observe a markedly higher seizure rate in infants $(45 \%$ in monitored children under 14 months compared to $18 \%$ in older children), the dearth of children between 12 and 20 months in our cohort does not allow us to determine how steep the drop in the seizure rate is as a function of age.

\section{Future Research}

Our current work raises new questions that should be addressed by future studies. Other than previous seizures, we were unable to detect any significant risk factors in older children. For comparison, in adults certain neurological conditions are associated with higher risk, but this effect may be less salient in young children (24). Guided by these insights, we conducted a simple power analysis which indicated that our study would reliably detect conditions with associated seizure risk above $50 \%$, if there were any. However, to detect the effect size corresponding to a risk factor associated with seizure risk of $30 \%$ (as opposed to the standard $18 \%$ baseline risk), we would need as many as 1,500 patients to ensure the statistical power of 0.8 (a conventionallyassumed value in the study design). Due to the small number of patients, we also did not explore the effect of certain conditions listed in the Materials and Methods section. Therefore, these conditions and their impact on seizure risk also require further investigation.

\section{CONCLUSIONS}

In this study, which is the largest single-center retrospective review of pediatric patients receiving cEEG monitoring to-date, we observe an extremely high incidence of seizures in criticallyill children with neurological conditions under 14 months. In

\section{REFERENCES}

1. Sanchez SM, Carpenter J, Chapman KE, Dlugos DJ, Gallentine WB, Giza CC, et al. Pediatric ICU EEG monitoring: current resources and practice in the United States and Canada. J Clin Neurophysiol. (2013) 30:156-60. doi: 10.1097/WNP.0b013e31827eda27

2. Abend N, Gutierrez-Colina A, Topjian A, Zhao H, Guo R, Donnelly M, et al. Nonconvulsive seizures are common in critically ill children. Neurology (2011) 76:1071-7. doi: 10.1212/WNL.0b013e318211c19e these children, the following factors are associated with an increased risk of seizures: intracranial hemorrhage, hypoxicischemic encephalopathy, and meningitis or encephalitis. Under the current practice, many of these children who do not undergo cEEG monitoring may suffer from non-convulsive seizures.

The study highlights the extraordinary vulnerability of the infant brain to the occurrence of acute symptomatic seizures. For providers who care for young patients, this special vulnerability should be reflected in their vigilance to and management of these patients and their problems.

\section{ETHICS STATEMENT}

The Institutional Review Board of Children's Healthcare of Atlanta approved this study (IRB protocol number 13-009). For this type of study, formal human participant consent was not required.

\section{AUTHOR CONTRIBUTIONS}

JV collected and analyzed data and wrote and edited the manuscript. MJ and QL performed data collection and analysis. TA and PK supervised and mentored JV, MJ, and QL assisted with data analysis and edited and revised the manuscript. JS was involved in study design, and manuscript revision. LO initiated the study, collected the data, and supervised all aspects of the study and manuscript preparation. AV is the primary mentor and coordinator for this study, provided oversight for all participants and reviewed and revised the manuscript.

\section{ACKNOWLEDGMENTS}

VPS data was provided by Virtual Pediatric Systems, LLC (VPS). No endorsement or editorial restriction of the interpretation of these data or opinions of the authors has been implied or stated.

We are also grateful to Ashley Sweeney BA, RN for serving as the VPS Site Coordinator at Children's.

\section{SUPPLEMENTARY MATERIAL}

The Supplementary Material for this article can be found online at: https://www.frontiersin.org/articles/10.3389/fped. 2018.00303/full\#supplementary-material

3. Topjian A, Gutierrez-Colina A, Sanchez S, Berg R, Friess S, Dlugos D, et al. Electrographic status epilepticus is associated with mortality and worse short-term outcome in critically ill children. Crit Care Med. (2013) 41:210. doi: 10.1097/CCM.0b013e31826 68035

4. Payne E, Zhao XY, Frndova H, McBain K, Sharma R, Hutchison J, et al. Seizure burden is independently associated with short term outcome in critically ill children. Brain (2014) 137 (Pt 5):1429-38. doi: 10.1093/brain/ awu042 
5. Herman $\mathrm{S}$, Abend $\mathrm{N}$, Bleck $\mathrm{T}$, Chapman $\mathrm{K}$, Drislane $\mathrm{F}$, Emerson R, et al. Consensus statement on continuous EEG in critically ill adults and children, part I: indications. $J$ Clin Neurophysiol. (2015) 32:87-95. doi: 10.1097/WNP.00000000000 00166

6. Greiner HM, Holland K, Leach JL, Horn PS, Hershey AD, Rose DF. Nonconvulsive status epilepticus: the encephalopathic pediatric patient. Pediatrics (2012) 129:e748-55. doi: 10.1542/peds.2011-2067

7. Hyllienmark L, Åmark P. Continuous EEG monitoring in a paediatric intensive care unit. Eur J Paediatr Neurol. (2007) 11:70-5. doi: 10.1016/j.ejpn.2006.11.005

8. McCoy B, Sharma R, Ochi A, Go C, Otsubo H, Hutchison JS, et al. Predictors of nonconvulsive seizures among critically ill children. Epilepsia (2011) 52:1973-8. doi: 10.1111/j.1528-1167.2011.03291.x

9. Williams K, Jarrar R, Buchhalter J. Continuous video-EEG monitoring in pediatric intensive care units. Epilepsia (2011) 52:1130-6. doi: 10.1111/j.1528-1167.2011.03070.x

10. Jette N, Claassen J, Emerson R, Hirsch L. Frequency and predictors of nonconvulsive seizures during continuous electroencephalographic monitoring in critically ill children. Arch Neurol. (2006) 63:1750-5. doi: 10.1001/archneur.63.12.1750

11. Shahwan A, Bailey C, Shekerdemian L, Harvey S. The prevalence of seizures in comatose children in the pediatric intensive care unit: a prospective video-EEG study. Epilepsia (2010) 51:1198-204. doi: 10.1111/j.1528-1167.2009.02517.x

12. Kirkham F, Wade A, McElduff F, Boyd S, Tasker R, Edwards M, et al. Seizures in 204 comatose children: incidence and outcome. Intens Care Med. (2012) 38:853-62. doi: 10.1007/s00134-012-2529-9

13. Abend NS, Arndt DH, Carpenter JL, Chapman KE, Cornett KM, Gallentine WB, et al. Electrographic seizures in pediatric ICU patients Cohort study of risk factors and mortality. Neurology (2013) 81:383-91. doi: 10.1212/WNL.0b013e31829c5cfe

14. Shellhaas RA, Chang T, Tsuchida T, Scher MS, Riviello JJ, Abend NS, et al. The American Clinical Neurophysiology Society's guideline on continuous electroencephalography monitoring in neonates. J Clin Neurophysiol. (2011) 28:611-7. doi: 10.1097/WNP.0b013e31823e96d7

15. Yang A, Arndt DH, Berg RA, Carpenter JL, Chapman KE, Dlugos DJ, et al. Development and validation of a seizure prediction model in critically ill children. Seizure (2015) 25:104-11. doi: 10.1016/j.seizure.2014.09.013
16. VPS LLC. Virtual Pediatric Systems. Los Angeles, CA: LLC (2015).

17. R Core Team. R: A Language and Environment for Statistical Computing. Vienna: $\mathrm{R}$ Foundation for Statistical Computing (2014).

18. Ross GJ, Tasoulis DK, Adams NM. Sequential monitoring of a Bernoulli sequence when the pre-change parameter is unknown. Comput Stat. (2013) 28:463-79. doi: 10.1007/s00180-012-0311-7

19. Agresti A. Categorical Data Analysis. Hoboken, NJ: John Wiley \& Sons (2002).

20. Steyerberg EW, Eijkemans MJC, Harrell FE, Habbema JDF. Prognostic modelling with logistic regression analysis: a comparison of selection and estimation methods in small data sets. Stat Med. (2000) 19:1059-79. doi: 10. 1002/(sici)1097-0258(20000430)19:8<1059::aid-sim412>3.3.co;2-s

21. Harrell F. Regression Modeling Strategies: With Applications to Linear Models, Logistic and Ordinal Regression, and Survival Analysis. Vol. 25. 2nd ed. Switzerland: Springer International Publishing (2015). 582 p.

22. Huang J, Gretton A, Borgwardt KM, Schölkopf B, Smola AJ. Correcting sample selection bias by unlabeled data. Adv Neural Info Process Syst. (2006) 19:601-8.

23. LaRoche S. Handbook of ICU EEG Monitoring. New York: Demos Medical Publishing (2012). 352 p.

24. Claassen J, Taccone F, Horn P, Holtkamp M, Stocchetti N, Oddo M. Recommendations on the use of EEG monitoring in critically ill patients: consensus statement from the neurointensive care section of the ESICM. Intens Care Med. (2013) 39:1337-51. doi: 10.1007/s00134-0132938-4

Conflict of Interest Statement: The authors declare that the research was conducted in the absence of any commercial or financial relationships that could be construed as a potential conflict of interest.

Copyright (C) 2018 Vlachy, Jo, Li, Ayer, Keskinocak, Swann, Olson and Vats. This is an open-access article distributed under the terms of the Creative Commons Attribution License (CC BY). The use, distribution or reproduction in other forums is permitted, provided the original author(s) and the copyright owner(s) are credited and that the original publication in this journal is cited, in accordance with accepted academic practice. No use, distribution or reproduction is permitted which does not comply with these terms. 